\title{
Next-Generation Techniques for Discovering Human Monoclonal Antibodies
}

\author{
A. A. Lushova ${ }^{a}$, M. G. Biazrova ${ }^{a}$, A. G. Prilipov ${ }^{b}$, G. K. Sadykova ${ }^{b}$, T. A. Kopylov ${ }^{c}$, and A. V. Filatov ${ }^{a}$ * \\ ${ }^{a}$ Institute of Immunology, Federal Medical-Biological Agency of Russia, Moscow, 115478 Russia \\ ${ }^{b}$ Ivanovsky Institute of Virology, Gamaleya Scientific Research Institute of Epidemiology and Microbiology, \\ Ministry of Health of the Russian Federation, Moscow, 123098 Russia \\ ${ }^{c}$ Orekhovich Institute of Biomedical Chemistry, Moscow, 119121 Russia \\ *e-mail:avfilat@yandex.ru
}

Received April 15, 2017; in final form, May 12, 2017

\begin{abstract}
Monoclonal antibodies have found wide applications in the treatment of cancer, as well as of autoimmune, infectious, and other diseases. Several dozen new antibodies are currently undergoing different stages of clinical trials, and some of them will soon be added to the list of immunotherapeutic drugs. Most of these antibodies have been generated using hybridoma technology or a phage display. In recent years, new methods of obtaining human monoclonal antibodies have been actively developing. These methods rely on sequencing immunoglobulin genes from B lymphocytes, as well as on the creation of antibody-secreting stable B-cell lines. The term next-generation antibody-discovery platforms has already been established in the literature to refer to these approaches. Our review focuses on describing the results obtained by these methods.
\end{abstract}

Keywords: human monoclonal antibodies, next-generation sequencing, B-cell immortalization, immunoglobulins

DOI: $10.1134 / \mathrm{S} 0026893317060103$

\section{INTRODUCTION}

Important recent advances in biomedical science have been associated with development and clinical application of biological agents based on monoclonal antibodies $(\mathrm{mAb})$. Antibodies can be used in immunotherapy to specifically activate or inhibit certain cell functions, to perform targeted delivery of chemotherapeutic agents to tumor cells, to neutralize cytokines, or to deactivate viruses. In this context, development and refinement of various methods of human $\mathrm{mAb}$ isolation becomes an issue of central importance.

For the first time, mAbs were obtained by Köhler and Milstein using the hybridoma technique [1]. Initially, this method could be used to obtain only mouse antibodies. With the development of transgenic mouse strains carrying human immunoglobulin genes [2], it became possible to obtain purely human antibodies. Despite being a rather old invention, the hybridoma technique remains the principal method of $\mathrm{mAb}$ production. However, this method has certain important shortcomings, the most significant of which is the low probability of hybridization between partner cells $\left(10^{-4}\right.$ to $\left.10^{-5}\right)$. As a result, the efficiency of obtaining

Abbreviations: ELISpot, enzyme-linked immunospot; NGS, next-generation sequencing; $\mathrm{VH}$, variable domain of the heavy chain; VL, variable domain of the light chain; mAb, monoclonal antibody; SARS, severe acute respiratory syndrome. hybridomas for weakly immunogenic proteins is poor. In addition, the use of transgenic mice carrying Ig genes is subject to strict regulation and rather expensive.

An alternative approach to $\mathrm{mAb}$ isolation is represented by various combinatorial techniques, including phage, DNA, RNA, and yeast displays, which have been in use since the 1990s. They are based on the analysis of vast $I g$ gene libraries. The most popular technique, phage display, has already been successfully employed to obtain several mAbs used in clinical immunotherapy, in particular, adalimumab, which inhibits the binding of tumor necrosis factor to its receptor [3]. However, compared to the hybridoma technology, this approach has been just moderately successful. The principal shortcoming of the combinatorial methods is that the resulting antibodies do not undergo selection in vivo. Furthermore, this method frequently generates antibodies with nonphysiological pairing of Ig chains, i.e., a pairing that never occurs in natural immune response. From this point of view, agents obtained using combinatorial techniques can be considered as antibody mimetics, which are likely to induce the production of neutralizing antibodies in patients during long-term administration [4, 5].

Thus, traditional approaches based on the hybridoma method and combinatorial techniques have considerable shortcomings, and there is a need for new, better methods of obtaining human mAbs. This review 
Table 1. Characterization of B cells used as a source of immunoglobulin genes

\begin{tabular}{l|l|l}
\hline \multicolumn{1}{c|}{ Ig gene source } & \multicolumn{1}{c}{ Phenotype } & \multicolumn{1}{c}{ Localization } \\
\hline Plasma cells & High mRNA level, Ig secretion, $\mathrm{BCR}^{-}, \mathrm{CD} 138^{\mathrm{hi}}$ & Bone marrow \\
Memory B cells & $\mathrm{CD} 19^{+}, \mathrm{CD} 20^{+}, \mathrm{CD} 27^{+}, \mathrm{IgG}^{+}, \mathrm{BCR}^{+}$ & Blood, day 14-20 after immunization \\
Plasmablasts & $\mathrm{CD} 19^{+}, \mathrm{CD} 20^{\text {low }}, \mathrm{CD} 27^{\mathrm{hi}}, \mathrm{CD}^{\mathrm{hi}}, \mathrm{BCR}^{+}$ & Blood, day 7 after immunization \\
\hline
\end{tabular}

focuses on describing new approaches to mAb discovery based on direct sequencing of B-cell Ig genes, as well as on the creation of immortal B-cell clones. A term has already been coined for these techniques; next-generation antibody discovery platforms [6].

\section{APPLICATION OF NEXT-GENERATION SEQUENCING TECHNIQUES FOR IMMUNOGLOBULIN GENE CLONING}

The advent of the next-generation sequencing (NGS) techniques and the progress in the bioinformatic data analysis have created unprecedented possibilities in genome research. NGS technique enables the simultaneous reading of up to several million variants of a single gene, which is especially important for the highly variable $I g$ genes. The repertoire of the CDR-H3 domain (the third complementarity-determining region of the antibody heavy chain) alone may include $10^{6}$ to $10^{7}$ clonotypes [7]. Obviously, the repertoire of $V H: V L$ clonotypes is even more diverse. Several cases of the NGS application for mAb production have already been reported [8].

From the bone marrow of mice immunized with ovalbumin, the pool of CD45 $\mathrm{R}^{-} \mathrm{CD} 138^{+}$plasma cells was isolated and analyzed by NGS [9]. The resulting $V L$ and $V H$ repertoire included several hundred thousand variants. It should be noted that up to $20 \%$ of the $\mathrm{CDR}-\mathrm{H3}$ repertoire was represented by only four sequences. This led to the hypothesis that the most frequently occurring sequences corresponded to antigen-specific Ig. Combinations of the most represented heavy and light chain sequences were expressed, and clones that secrete antigen-specific antibodies were identified by screening.

Ig genes are characterized by high mutation frequencies; therefore, the reliable identification of specific sequences requires that the whole variable region be read through completely. For this purpose, the read length should be increased to at least $480 \mathrm{bp}$, which is in fact a threshold capacity level of modern NGS analyzers. However, advances in the development of new equipment inspire certain hope. Simultaneous progress in software development should also contribute to solving this complex problem $[10,11]$. The quality of reading can be significantly improved by employing unique molecular identification tags (bar codes) [12, 13], which serve to distinguish between PCR artifacts and rare mutations in the variable $I g$ gene region.
Wu et al. used NSG to sequence a cDNA library obtained from antigen-specific B cells isolated from the blood of an HIV-infected donor [14]. First, nucleotide sequences that encode the known broadly neutralizing antibodies VRC-01-VRC-03 were used to deduce their potential precursor gene. In a cDNA library of several thousand sequences, those that originated from a common embryonic line precursor were identified. From this group, approximately 5000 variants with the highest resemblance to VRC-01-VRC-03 were selected. Among 45 randomly chosen and expressed mAbs, 24 mAbs exhibited broad-range neutralizing activity. These results indicate that NGS is applicable for discovering novel mAbs.

\section{IMMUNOGLOBULIN GENE AMPLIFICATION FROM INDIVIDUAL B CELLS}

The principal shortcoming of the NGS-based approach is the loss of information about "physiological" pairing of heavy and light Ig chains. To overcome this limitation, it was proposed to amplify, clone, and express antibodies from individual B cells [15]. Potential sources of $I g$ genes are plasma cells, plasmablasts, or memory B cells (Table 1$)$.

\section{PLASMA CELLS AS A SOURCE OF IMMUNOGLOBULIN GENES}

Plasma cells are considered to be the most appropriate object for Ig gene sequencing, since they extensively synthesize antibodies and, thus, contain large amounts of corresponding mRNAs. This circumstance facilitates the procedure for cDNA amplification; however, plasma cells do not carry surface B-cell receptors and, for this reason, cannot be isolated by antigen-specific selection. The fact that plasma cells are located in the bone marrow also represents a significant limitation to experiments.

In a study by Clargo et al. [16], plasma cells were isolated from the bone marrow of rabbits immunized with a human tumor necrosis factor receptor. For this purpose, bone marrow cells were placed into gel growth medium that contained antigen-loaded particles, as well as fluorescein-labeled secondary antibodies against the constant fragment of rabbit Ig. Fluorescent microscopy of these preparations revealed that each antigen-producing $\mathrm{B}$ cell was surrounded by a fluorescent halo, which was formed as a result of anti- 
body binding to the particles in direct vicinity of producing plasma cells.

As a result, out of nearly 4 million bone marrow cells, 96 antigen-specific plasma cells were isolated and 50 recombinant antibodies with endogenous $\mathrm{VH}-$ VL pairing were obtained; six of these antibodies exhibited a high level of blocking activity, preventing the binding of tumor necrosis factor to its receptor.

A study by Corti et al. [17] had the goal of obtaining broadly neutralizing antibodies against influenza virus. For this purpose, individual CD $138^{+}$plasma cells were isolated from the blood of vaccinated donors. Stimulation with IL-6 allowed plasma cells to survive for several days, which made it possible to screen for the secreted antibodies against a broad panel of virus subtypes. By sequencing Ig genes of the selected cells, the authors were able to obtain broadly neutralizing antibodies against influenza virus.

\section{MEMORY B CELLS AS A SOURCE OF IMMUNOGLOBULIN GENES}

In contrast to plasma cells, memory $\mathrm{B}$ cells express B-cell receptors on their surface and can be sorted and isolated using the corresponding antigens. However, starting on days 14-20 after final immunization, the blood levels of memory B cells begin to decrease, which makes them more difficult to detect and isolate, if more time has passed after immunization or infection.

To increase the abundance of memory B cells, they can be transformed by the Epstein-Barr virus and subsequently cultivated. A particularly impressive example of using this approach is the study by Yu et al. [18], who obtained human $\mathrm{mAb}$ against the influenza virus A (H1N1) strain associated with the Spanish flu pandemics. In 2008, memory B cells were isolated from donors who had influenza in 1918; the cells were immortalized and cultivated, after which Ig genes were sequenced and expressed. It can be estimated that, 90 years after the infection, memory $\mathrm{B}$ cells were present in donors with a frequency of 1 cell per $4.6 \times 10^{6} \mathrm{~B}$ cells; nevertheless, despite this low occurrence, the authors were able to obtain antigen-specific antibodies.

Difficulties that arise in B-cell immortalization induced by Epstein-Barr virus can be partially overcome by means of preliminary lymphocyte activation using CpG-containing oligonucleotides. It was by this approach that $\mathrm{S} 3.1 \mathrm{mAb}$ were obtained using memory $\mathrm{B}$ cells from the blood of a patient who had recovered from severe acute respiratory syndrome (SARS). These mAbs had a protective effect in a murine model of infection caused by SARS-CoV coronavirus [19].

Another example of mAb production using memory B cells was described by Scheid et al. [20]. Memory $B$ cells were isolated from the blood of six HIVinfected patients who possessed broadly neutralizing antibodies against the virus. Antigen-specific memory B cells were selected using their binding to a gp140 viral protein carrying a fluorescent label. After the corresponding $I g$ genes were sequenced and expressed, it was found that the immune response was mediated by at least 50 independent clones that targeted different regions of gp 120. Thus, although populations of memory B cells are small, they can nevertheless be used to obtain mAbs against the corresponding pathogen, even a long time after the infection.

\section{PLASMABLASTS AS A SOURCE OF IMMUNOGLOBULIN GENES}

The earliest antibody-producing cells are plasmablasts, precursors of memory B cells, and plasma cells. Normally, plasmablasts constitute a small portion of all circulating B cells (no more than $0.2 \%$ ), but their number increases dramatically during infection or after immunization. Plasmablast concentration in blood peaks on day 7 after immunization, amounting to approximately $6 \%$ of all peripheral B cells [21]. During an acute infection, the share of plasmablasts in the total number of circulating B cells may reach $30 \%$ [13]. Smith et al. [22] proposed a method that enabled them to obtain mAbs against influenza virus, anthrax toxin, and pneumococcal antigen using plasmablasts (Fig. 1).

According to this method, lymphocytes were isolated from donors' blood on day 7 after vaccination. The efficiency of immunization and the abundance of antigen-specific B cells were assessed using the enzyme-linked immunospot (ELISpot) technique. Flow cytometry was used to sort plasmablasts by phenotype, one cell per plate well. mRNA was isolated from these cells, and cDNA was synthesized by RTPCR and amplified by nested PCR. Following this algorithm, the authors were able to obtain an amount of DNA sufficient for the subsequent sequencing of $I g$ genes. RT-PCR was performed with nine primers to cover the whole family of $\mathrm{V}$ fragment genes. The resulting two PCR products contained sequences that encoded $\mathrm{H}$ - and L-chains and were cloned into expression vectors. These plasmids were used to transfect HEK293 cells and expressed to produce mAbs.

From $20 \mathrm{~mL}$ of donor blood, the authors were able to obtain 120 to 200 different mAbs. In particular, for antibodies against influenza virus, it was shown that nearly $40 \%$ of B cells from the whole pool of reactive plasmablasts originated from a single embryonic precursor.

Currently, the most appropriate method of cell sorting for subsequent cloning is flow cytometry; its important advantages include the high purity of the resulting cell population, as well as the possibility to sort individual cells, which is crucial for cloning purposes. Moreover, flow cytometry allows cells to be sorted using any combination of detectable parameters. By combining several selection parameters, it is possible to improve the specificity of isolation considerably. Plasmablasts are most commonly isolated based on the $\mathrm{IgG}^{+} \mathrm{IgA}^{-} \mathrm{IgM}^{-}$ $\mathrm{CD} 19^{+} \mathrm{CD} 20^{\text {low }} \mathrm{CD} 27^{\text {hi }} \mathrm{CD} 38^{\text {hi }}$ phenotype [13]. 


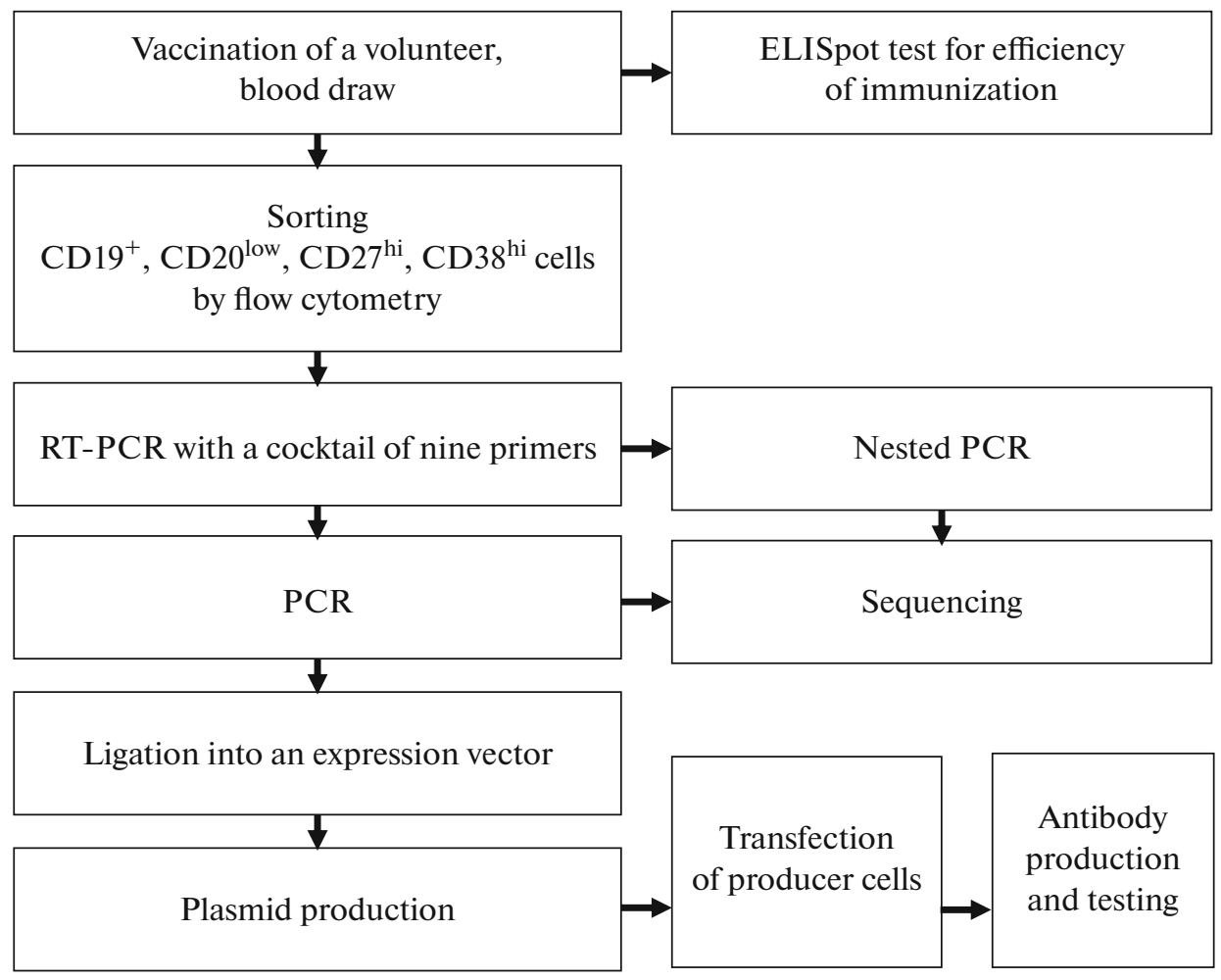

Fig. 1. Algorithm of mAb production by cloning $I g$ genes from individual plasmablasts as proposed by Smith et al. [22].

The task of simultaneously amplifying and sequencing two genes, $V H$ and $V L$, is associated with certain difficulties. To facilitate the procedure, it was proposed to fuse these genes early in the experiment and subsequently treat them as a single fusion gene [23]. In order to conveniently express variable $I g$ sequences, Liao et al. developed a special cassette containing all necessary elements for gene transcription and the regulation of their translation, including a cytomegalovirus promoter, an Ig-leader peptide, constant fragments of light and heavy Ig chains, and an appropriate polyA sequence [24].

For ethical reasons, the possibility to immunize humans with certain antigens is strongly limited. In these cases, immunodeficient SCID/beige mice can be used as temporal hosts for human B cells [25, 26]. Human B cells were inoculated into the spleen of sublethally irradiated mice, after which the animals were immunized. Irradiation served to achieve complete depletion of lymphatic organs in order to enable sub-

Table 2. Comparison of NGS and single-cell RT-PCR of Ig genes

\begin{tabular}{l|l|l}
\hline \multicolumn{1}{c|}{ Parameter } & NGS & $\begin{array}{l}\text { Single-cell } \\
\text { RT-PCR }\end{array}$ \\
\hline Throughput & High & Low \\
H and L chain pairing & No & Yes \\
Determining the size of clonal families & Yes & No \\
\hline
\end{tabular}

sequent lymphopenic proliferation of the inoculated lymphocytes within free niches of the spleen. On day 8 after lymphocyte transplantation, the portion of human plasmablasts was $40 \%$ and higher. Using this method, the authors obtained broadly neutralizing antibodies against influenza virus.

\section{COMBINING THE TECHNIQUES OF NGS AND SINGLE-CELL IG GENE RT-PCR}

As was mentioned above, the high-throughput sequencing of Ig genes derived from a pool of B cells does not take into account the physiological pairing of $\mathrm{VH}$ and VL chains, which prevents the functional characterization of endogenous antibodies. Furthermore, it is fairly difficult to determine how the number of sequences obtained by NGS is related to the number of cells that express the corresponding gene sequences to secrete antibodies. A comparison of advantages and shortcomings of NGS and single-cell Ig gene sequencing (Table 2) suggests that it should be promising to combine both of these techniques in the same experiment.

A study by Tan et al. [27] demonstrated the possibilities of combining the two approaches. B cells of volunteer donors vaccinated against influenza virus were sorted into plate wells, one cell per well. During reverse transcription, cDNA was labeled with bar code sequences specific to each well. Following PCR, specimens were combined and analyzed using NGS. 
Those $V H$ and $V L$ sequences that carried the same bar code corresponded to the physiologically correct pairing of the light and the heavy Ig chain. mAbs against influenza virus antigens were obtained based on the expression of the most frequently occurring physiological pairs of $I g$ gene sequences.

A more sophisticated approach was employed by DeKosky et al. [28], who used the so-called paired $V H-V L$ analysis. Blood B cells were sorted by one per well using flow cytometry. Each well also contained paramagnetic particles carrying poly(dT) molecules for mRNA binding. Following cell lysis, particles from different wells were combined, and emulsion RT-PCR was performed so as to amplify cDNA from each particle in an individual drop of emulsion. Amplification was performed with overlapping primers in order to obtain a single PCR product containing gene sequences for the heavy and light Ig chains fused via a short linker. In this way, it was possible to detect the expression of $I g$ genes of antibodies broadly presented in the immunological repertoire, as well as those that correspond to rare B-cell clones. If the sequence data obtained by NGS overlap insufficiently, mass spectrometry-based proteomic methods can be additionally employed to determine amino acid sequences of variable Ig domains [29, 30].

\section{PRODUCTION OF MONOCLONAL ANTIBODIES IN STABLE CULTURES OF PROLIFERATING B CELLS}

The most obvious alternative to $I g$ gene sequencing for the purposes of mAb production might be the immortalization of B cells with their subsequent cloning and cultivation. Indeed, this approach has long proved efficient for obtaining antigen-specific $T$ cell clones. However, the seemingly obvious experiments on B-cell immortalization encountered serious difficulties that appeared unsurmountable for a long time. The first attempts to obtain constantly growing B-cell clones were based on their transformation with Epstein-Barr virus. However, these cells exhibited limited growth potential, low cloning efficiency, and insufficient Ig production levels that rapidly decreased during cultivation [31-34].

A broad range of various activators have been applied to stimulate the growth of B cells, ranging from different interleukins [35] to TLR7/TLR8 Toll-like receptor ligands, $\mathrm{CpG}$-containing oligonucleotides, and Staphylococcus aureus [36]. With all of the diversity of approaches tested, no eternal youth elixir could be found for B cells so far, although it was determined that, in order to maintain B cell division, it is essential that feeder cells express CD40 ligand [37, 38]. However, the more efficient the B-cell stimulation, the more rapidly they underwent terminal differentiation into plasma cells incapable of further growth.

An important breakthrough was a study in which the stimulation of B cells was combined with their reprogramming due to the expression of BCL-6 and BCL-XL transcription factors [39]. BCL-6 served to keep B cells in the plasmablast state, while BCL-XL prevented them from entering apoptosis. Under these conditions, the plasmablast population increased by more than 100 times during 12 days of cultivation. The resulting B-cell clones could be screened and used to produce the required antibodies. The described technique was successfully applied to obtain mAbs against Clostridium tetani, influenza virus, respiratory syncytial virus, and parechovirus [40].

Dividing plasmablasts express activation-induced cytidine deaminase, an enzyme involved in somatic hypermutagenesis that occurs during affinity maturation. Flow cytometry and an antigen carrying a fluorescent label were used to select lymphocytes with the highest affinity to the antigen from the total pool of antigen-specific $B$ cells and to obtain antibodies, the affinity of which was an order of magnitude higher than in the initial population [41]. This approach provides new possibilities not only to obtain mAbs, but also to improve them.

Thus, the recent research has produced some novel methods of mAb discovery, the number of which is constantly growing. We have every reason to believe that, in the nearest future, these and other innovative approaches will come to play a central role in the development of novel drugs for the immunotherapy of different human diseases.

\section{ACKNOWLEDGMENTS}

This work was supported by the Russian Science Foundation grant no. 17-75-20106.

\section{REFERENCES}

1. Köhler G., Milstein C. 1975. Continuous cultures of fused cells secreting antibody of predefined specificity. Nature. 256, 495-497.

2. Green L.L. 1999. Antibody engineering via genetic engineering of the mouse: XenoMouse strains are a vehicle for the facile generation of therapeutic human monoclonal antibodies. J. Immunol. Methods. 231, 11-23.

3. Frenzel A., Schirrmann T., Hust M. 2016. Phage display-derived human antibodies in clinical development and therapy. MAbs. 8, 1177-11194.

4. Chiu H.Y., Wang T.S., Chan C.C., et al. 2015. Risk factor analysis for the immunogenicity of adalimumab associated with decreased clinical response in Chinese patients with psoriasis. Acta Dermatol. Venereol. 95, 711-716.

5. van Schouwenburg P.A., Rispens T., Wolbink G.J. 2013. Immunogenicity of anti-TNF biologic therapies for rheumatoid arthritis. Nat. Rev. Rheumatol. 9, 164-172.

6. Hardiman G. 2012. Next-generation antibody discovery platforms. Proc. Natl. Acad. Sci. U. S. A. 109, 18245-18246.

7. Lavinder J.J., Horton A.P., Georgiou G., et al. 2015. Next-generation sequencing and protein mass spectrometry for the comprehensive analysis of human cellular and serum antibody repertoires. Curr. Opin. Chem. Biol. 24, 112-120. 
8. Galson J.D., Pollard A.J., Truck J., et al. 2014. Studying the antibody repertoire after vaccination: Practical applications. Trends Immunol. 35, 319-331.

9. Reddy S.T., Ge X., Miklos A.E., et al. 2010. Monoclonal antibodies isolated without screening by analyzing the variable-gene repertoire of plasma cells. Nat. Biotechnol. 28, 965-969.

10. Lees W.D., Shepherd A.J. 2015. Utilities for highthroughput analysis of B-cell clonal lneages. J. Immunol. Res. 2015, 323-506.

11. Lebedin M.Y., Turchaninova M.A., Egorov E.S., et al. 2017. High-throughput immunoglobulin sequencing data analysis with the use of unique molecular identifiers. Immunologiya. 38, 59-63.

12. Turchaninova M.A., Davydov A., Britanova O.V., et al. 2016. High-quality full-length immunoglobulin profiling with unique molecular barcoding. Nat. Protoc. 11, 1599-1616.

13. Lu D.R., Tan Y.C., Kongpachith S., et al. 2014. Identifying functional anti-Staphylococcus aureus antibodies by sequencing antibody repertoires of patient plasmablasts. Clin. Immunol. 152, 77-89.

14. Wu X., Zhou T., Zhu J., Zhang B., et al. 2011. Focused evolution of HIV-1 neutralizing antibodies revealed by structures and deep sequencing. Science. 333, 1593-1602.

15. Wardemann H., Yurasov S., Schaefer A., et al. 2003. Predominant autoantibody production by early human B cell precursors. Science. 301, 1374-1377.

16. Clargo A.M., Hudson A.R., Ndlovu W., et al. 2014. The rapid generation of recombinant functional monoclonal antibodies from individual, antigen-specific bone marrow-derived plasma cells isolated using a novel fluorescence-based method. MAbs. 6, 143-159.

17. Corti D., Voss J., Gamblin S.J., Codoni G., et al. 2011. A neutralizing antibody selected from plasma cells that binds to group 1 and group 2 influenza A hemagglutinins. Science. 333, 850-856.

18. Yu X., Tsibane T., McGraw P.A., House F.S., et al. 2008. Neutralizing antibodies derived from the B cells of 1918 influenza pandemic survivors. Nature. 455, 532-536.

19. Traggiai E., Becker S., Subbarao K., Kolesnikova L., et al. 2004. An efficient method to make human monoclonal antibodies from memory B cells: Potent neutralization of SARS coronavirus. Nat. Med. 10, 871-875.

20. Scheid J.F., Mouquet H., Feldhahn N., et al. 2009. Broad diversity of neutralizing antibodies isolated from memory B cells in HIV-infected individuals. Nature. 458, 636-640.

21. Wrammert J., Smith K., Miller J., et al. 2008. Rapid cloning of high-affinity human monoclonal antibodies against influenza virus. Nature. 453, 667-671.

22. Smith K., Garman L., Wrammert J., et al. 2009. Rapid generation of fully human monoclonal antibodies specific to a vaccinating antigen. Nat. Protoc. 4, 372-384.

23. Tiller T., Meffre E., Yurasov S., et al. 2008. Efficient generation of monoclonal antibodies from single human B cells by single cell RT-PCR and expression vector cloning. J. Immunol. Methods. 329, 112-124.

24. Liao H.X., Levesque M.C., Nagel A., et al. 2009. Highthroughput isolation of immunoglobulin genes from single human B cells and expression as monoclonal antibodies. J. Virol. Methods. 158, 171-179.
25. Lin Z., Chiang N.Y., Chai N., et al. 2014. In vivo antigen-driven plasmablast enrichment in combination with antigen-specific cell sorting to facilitate the isolation of rare monoclonal antibodies from human B cells. Nat. Protoc. 9, 1563-1577.

26. Nakamura G., Chai N., Park S., et al. 2013. An in vivo human-plasmablast enrichment technique allows rapid identification of therapeutic influenza A antibodies. Cell Host Microbe. 14, 93-103.

27. Tan Y.C., Blum L.K., Kongpachith S., et al. 2014. High-throughput sequencing of natively paired antibody chains provides evidence for original antigenic sin shaping the antibody response to influenza vaccination. Clin. Immunol. 151, 55-65.

28. DeKosky B.J., Ippolito G.C., Deschner R.P., et al. 2013. High-throughput sequencing of the paired human immunoglobulin heavy and light chain repertoire. Nat. Biotechnol. 31, 166-169.

29. Wine Y., Boutz D.R., Lavinder J.J., et al. 2013. Molecular deconvolution of the monoclonal antibodies that comprise the polyclonal serum response. Proc. Natl. Acad. Sci. U. S. A. 110, 2993-2998.

30. Cheung W.C., Beausoleil S.A., Zhang X., et al. 2012. A proteomics approach for the identification and cloning of monoclonal antibodies from serum. Nat. Biotechnol. 30, 447-452.

31. Aman P., Ehlin-Henriksson B., Klein G. 1984. Epstein-Barr virus susceptibility of normal human B lymphocyte populations. J. Exp. Med. 159, 208-220.

32. Crain M.J., Sanders S.K., Butler J.L., et al. 1989. Epstein-Barr virus preferentially induces proliferation of primed B cells. J. Immunol. 143, 1543-1548.

33. Laffly E., Sodoyer R. 2005. Monoclonal and recombinant antibodies, 30 years after. Hum. Antibodies. 14, 33-55.

34. Stahli C., Staehelin T., Miggiano V., et al. 1980. High frequencies of antigen-specific hybridomas: Dependence on immunization parameters and prediction by spleen cell analysis. J. Immunol. Methods. 32, 297-304.

35. Banchereau J., Bazan F., Blanchard D., et al. 1994. The CD40 antigen and its ligand. Annu. Rev. Immunol. 12, 881-922.

36. Banchereau J., Rousset F. 1991. Growing human B lymphocytes in the CD40 system. Nature. 353, 678-679.

37. O’Nions J., Allday M.J. 2004. Proliferation and differentiation in isogenic populations of peripheral B cells activated by Epstein-Barr virus or T cell-derived mitogens. J. Gen. Virol. 85, 881-895.

38. Wiesner M., Zentz C., Mayr C., et al. 2008. Conditional immortalization of human B cells by CD40 ligation. PLoS ONE. 3, e1464.

39. Kwakkenbos M.J., Diehl S.A., Yasuda E., et al. 2010. Generation of stable monoclonal antibody-producing B cell receptor-positive human memory B cells by genetic programming. Nat. Med. 16, 123-128.

40. Kwakkenbos M.J., van Helden P.M., Beaumont T., et al. 2016. Stable long-term cultures of self-renewing B cells and their applications. Immunol. Rev. 270, 65-77.

41. Kwakkenbos M.J., Bakker A.Q., van Helden P.M., et al. 2014. Genetic manipulation of B cells for the isolation of rare therapeutic antibodies from the human repertoire. Methods. 65, 38-43.

Translated by D. Timchenko 